\title{
ARTICLE
}

Clinical Research

\section{A higher BMI is not associated with a different immune response and disease course in critically ill COVID-19 patients}

\author{
Emma J. Kooistra $\mathbb{1}^{1,2}$ - Aline H. de Nooijer ${ }^{2,3} \cdot$ Wout J. Claassen ${ }^{1,2}$ - Inge Grondman ${ }^{2,3}$ • Nico A. F. Janssen $\mathbb{1}^{2,3} \cdot$ \\ Mihai G. Netea $\mathbb{D}^{2,3}$. Frank L. van de Veerdonk ${ }^{2,3}$ • Johannes G. van der Hoeven ${ }^{1,2}$ • Matthijs Kox $\mathbb{D}^{1,2}$ • \\ Peter Pickkers $\mathbb{1}^{1,2} \cdot$ on behalf of the RCI-COVID-19 study group
}

Received: 6 July 2020 / Revised: 2 November 2020 / Accepted: 4 January 2021 / Published online: 25 January 2021

(c) The Author(s), under exclusive licence to Springer Nature Limited 2021

\begin{abstract}
Background/objectives Obesity appears to be an independent risk factor for ICU admission and a severe disease course in COVID-19 patients. An aberrant inflammatory response and impaired respiratory function have been suggested as underlying mechanisms. We investigated whether obesity is associated with differences in inflammatory, respiratory, and clinical outcome parameters in critically ill COVID-19 patients.

Subjects/methods Sixty-seven COVID-19 ICU patients were divided into obese (BMI $\geq 30 \mathrm{~kg} / \mathrm{m}^{2}, n=18,72 \%$ class I obesity, $28 \%$ class II obesity) and non-obese (BMI $\left.<30 \mathrm{~kg} / \mathrm{m}^{2}, n=49\right)$ groups. Concentrations of circulating interleukin (IL)-6, IL-8, IL-10, tumor necrosis factor alpha (TNF- $\alpha$ ), interferon gamma (IFN- $\gamma$ ), interferon gamma-induced protein (IP)10, monocyte chemoattractant protein (MCP)-1, and IL-1 receptor antagonist (RA) were determined from ICU admission until 10 days afterward, and routine laboratory and clinical parameters were collected.

Results BMI was $32.6[31.2-34.5]$ and 26.0 [24.4-27.7] kg/m² in the obese and non-obese group, respectively. Apart from temperature, which was significantly lower in obese patients $\left(38.1[36.9-38.9]\right.$ vs. $\left.38.7[38.0-39.5]{ }^{\circ} \mathrm{C}, p=0.02\right)$, there were no between-group differences on ICU admission. Plasma cytokine concentrations declined over time $(p<0.05$ for all), but no differences between obese and non-obese patients were observed. Also, BMI did not correlate with the cytokine response (IL-6 $r=0.09, p=0.61$, TNF- $\alpha r=0.03, p=0.99$, IP-10 $r=0.28, p=0.11$ ). The kinetics of clinical inflammatory parameters and respiratory mechanics were also similar in both groups. Finally, no differences in time on ventilator, ICU length of stay or 40-day mortality between obese and non-obese patients were apparent.

Conclusions In COVID-19 patients requiring mechanical ventilation in the ICU, a higher BMI is not related to a different immunological response, unfavorable respiratory mechanics, or impaired outcome.
\end{abstract}

Members of the RCI-COVID-19 study group are listed above Author contributions.

Supplementary information The online version contains supplementary material available at https://doi.org/10.1038/s41366021-00747-z.

Peter Pickkers

peter.pickkers@radboudumc.nl

1 Department of Intensive Care Medicine, Radboud University Medical Center, 6500HB Nijmegen, The Netherlands

2 Radboud Center for Infectious Diseases, Radboud University Medical Center, 6500HB Nijmegen, The Netherlands

3 Department of Internal Medicine, Radboud University Medical Center, 6500HB Nijmegen, the Netherlands

\section{Background}

The Coronavirus Disease 2019 (COVID-19) pandemic currently sweeps across the globe, leading to high morbidity and mortality. This infection, caused by Severe Acute Respiratory Syndrome Coronavirus 2 (SARS-CoV-2), can cause acute respiratory distress syndrome (ARDS) requiring mechanical ventilation in the intensive care unit (ICU). Age, male sex, and multiple comorbidities, including type 2 diabetes, hypertension, and coronary artery disease, were identified as risk factors related to severity of COVID-19 disease [1-4]. These comorbidities are more prevalent in the obese population than in patients with a normal body weight [5]. Also, the prevalence of obesity among COVID19 patients requiring mechanical ventilation was shown to be higher than expected when compared to controls with 
non COVID-19-related acute pulmonary diseases, and the need for mechanical ventilation increased with increasing body mass index (BMI) [6, 7]. Finally, mortality was shown to be significantly higher in overweight/obese COVID-19 patients compared to normal-weight patients [7]. Of interest, obesity itself is also recognized as an independent risk factor of severe pulmonary H1N1 influenza infection and mortality [8].

The underlying mechanisms of obesity as a factor contributing to COVID-19 disease severity are currently poorly understood, but various possible explanations have been proposed. For instance, in obese patients, pulmonary function may be restricted because of decreased functional residual capacity, resulting in lower blood oxygen levels [9]. In addition, the angiotensin converting enzyme 2 receptor, which is utilized by SARS-CoV-2 to enter cells, is highly expressed by adipocytes and expression levels were shown to be higher in adipocytes of patients with obesity and type 2 diabetes [10]. Therefore, it appears plausible that adipose tissue may serve as a reservoir for the virus, leading to more pronounced and sustained viral shedding, resulting in a perpetual inflammatory response and impaired outcome.

To date, it is not known whether obesity is only a risk factor for COVID-19 susceptibility and for the need of intensive care, or whether it also influences the outcome of the patients once admitted on ICUs. Previous studies in critically ill COVID-19 patients have yielded ambiguous results, with some reporting associations between lower BMI and mortality [11], whereas others showed increased mortality in (severely) obese patients [12, 13]. In the present study, we investigated associations between obesity and the immune response as well as the clinical course of SARS$\mathrm{CoV}-2$ infection in critically ill patients on the ICU of a tertiary university hospital. Elucidating these mechanisms may pave the way for the development of new treatment strategies for obese COVID-19 patients.

\section{Methods}

\section{Study design and participants}

In this prospective observational cohort study, all consecutive COVID-19 patients admitted to the ICU in the Radboud University Medical Center (Nijmegen, The Netherlands) between March 11 and April 27 were included. COVID-19 was diagnosed by a positive SARS-CoV-2 RT-PCR test in nasopharyngeal and throat swabs and/or by typical chest CT-scan findings. Patients with a pre-existing immunosuppressed status or other comorbidities that strongly influence prognosis were excluded. Patients were divided into an obese (BMI $\geq 30 \mathrm{~kg} / \mathrm{m}^{2}$ ) and non-obese
$\left(\mathrm{BMI}<30 \mathrm{~kg} / \mathrm{m}^{2}\right)$ group, according to the classification of obesity by the World Health Organisation [14]. Also, individual BMI values were correlated with circulating cytokine concentrations. The study was carried out in the Netherlands in accordance with the applicable rules concerning the review of research ethics committees and informed consent. All patients or legal representatives were informed about the study details and could decline to participate.

\section{Data collection}

Data were collected from the electronic patient files (EPIC, EPIC Systems Corporation, Verona, Wisconsin, USA) and recorded in the good clinical practice-compliant data management system Castor (Castor EDC, Amsterdam, the Netherlands). ICU admission day was designated as day 1 , and serial data were obtained for 10 consecutive days. Clinical outcomes (time on mechanical ventilation, ICU length of stay (LOS), and mortality) were recorded for 40 consecutive days. Serial values of mean arterial pressure, body temperature, and leukocyte differentiation as well as circulating levels of C-reactive protein (CRP), procalcitonin (PCT), D-dimer, ferritin, and cytokines (see below) were used to assess the inflammatory response to SARS-CoV-2 infection. Patients were actively cooled when they had a fever of $>40^{\circ} \mathrm{C}$. In these cases, body temperature was imputed as $40^{\circ} \mathrm{C}$. Positive end expiratory pressure (PEEP), tidal volume per kilograms ideal body weight $\left(\mathrm{TV}_{\mathrm{IBW}}\right)$ and $\mathrm{PaO}_{2} / \mathrm{FiO}_{2}$ ratio were used to assess the level of respiratory support required. Tidal volume was only recorded in patients on volume-controlled mechanical ventilation. Because the vast majority of patients were switched to pressure support ventilation after 4 days of ICU admission, $\mathrm{TV}_{\mathrm{IBW}}$ data were analyzed until this timepoint. Ideal body weight was calculated based on sex and height, using the formulas provided by the ARDS Network [15].

\section{Cytokine concentration measurements}

A baseline blood sample was obtained within the first $48 \mathrm{~h}$ following ICU admission and serial samples were collected every other day. Ethylenediaminetetraacetic acidanticoagulated blood was centrifuged $(2000 \times g, 10 \mathrm{~min}$, $4{ }^{\circ} \mathrm{C}$ ), after which plasma was stored at $-80^{\circ} \mathrm{C}$ until analysis. Concentrations of interleukin (IL)-6, IL-8, IL-10, tumor necrosis factor (TNF)- $\alpha$, interferon gamma (IFN- $\gamma$ ), IFN- $\gamma$-induced protein (IP)-10, monocyte chemoattractant protein (MCP)-1, and IL-1 receptor antagonist (IL-1RA) were determined in one batch using a Luminex assay (Milliplex, Millipore, Billerica, USA). The lower detection limit was $3.2 \mathrm{pg} / \mathrm{mL}$ for all cytokines. 


\section{Statistical analysis}

Statistical analysis was performed using SPSS 25 (IBM) and Graphpad Prism 8 software (GraphPad Software). Several variables were not measured daily; therefore, depending on the frequency of measurements, data were binned into 2 or 3 days using a custom script made in Rstudio v3.6.2 (www.r-project.org). Because of the relatively small group size, normality was not assumed and all data are displayed as median with interquartile range [IQR] or geometric mean with $95 \%$ confidence interval. Baseline characteristics were analyzed using chi-square tests and Mann-Whitney $U$ tests. Between-group differences over time were analyzed using linear mixed effects model analysis on log-transformed data followed by posthoc analyses using Sidak's multiple comparisons tests in all serially measured variables. Relationships between BMI and cytokines were analyzed using Spearman's correlation. Time on mechanical ventilation, ICU LOS, and mortality were analyzed using log-rank and chisquare tests. Patients who died in the hospital or those who were still in the ICU and/or receiving mechanical ventilation on day 40 were censored at day 41 for the analysis of time on mechanical ventilation and ICU LOS. For the mortality analysis, patients who were discharged alive from the hospital or were still in the ICU or hospital on day 40 were censored at day 41 .

\section{Results}

\section{Patient characteristics}

All 77 patients with proven COVID-19 consecutively admitted to the ICU of Radboud University Medical Center were assessed for study inclusion. Patients with a preexisting immunosuppressed status were excluded $(n=9$, see Supplementary Fig. 1), and one patient refused participation. The remaining study population $(n=67)$ was divided in obese ( $n=18$, of which $72 \%$ with class I obesity and $28 \%$ with class II obesity) and non-obese $(n=49)$ groups (Fig. 1a). BMI of the entire study population was 27.7 [24.9-30.8] kg/m² (Fig. 1), with BMI's of 32.6 [31.2-34.5] and 26.0 [24.4-27.7] $\mathrm{kg} / \mathrm{m}^{2}$ in the in the obese and non-obese groups, respectively $(p<0.0001$, Table 1$)$. All patients were mechanically ventilated. Temperature at ICU admission was 38.1 [36.9-38.9] ${ }^{\circ} \mathrm{C}$ in the obese group and 38.7 [38.0-39.5] ${ }^{\circ} \mathrm{C}$ in the non-obese group $(p=$ 0.007). No significant differences in age, sex, APACHE II score, medical history, and other clinical or laboratory parameters between the two groups were observed at ICU admission (Table 1).

\section{Inflammation and respiration}

Plasma concentrations of all measured pro-inflammatory cytokines were highest at ICU admission and decreased over time both in the obese and non-obese groups $(p<0.05$ for all cytokines, Fig. 1b-i). Except for slightly higher IP-10 levels in obese vs. non-obese patients at days 9-10, no between-group differences in any of the measured cytokines on any timepoints were observed (Fig. 1b-i). Furthermore, BMI did not correlate with concentrations of IL- 6 , TNF- $\alpha$, and IP-10 at ICU admission $(r=-0.09 p=0.61, r=0.03$ $p=0.99$ and $r=0.28 p=0.11$, respectively, Fig. 2) or any other cytokine measured ( $r$ values ranging from -0.11 to $0.06, p$ values all $>0.50$, see Supplementary Fig 2). Despite a trend toward less pronounced leucocytosis in obese patients, no significant differences were present between both groups at any of the timepoints (Fig. 3a). The baseline difference in temperature (i.e., higher in non-obese patients) persisted at later timepoints, but did not attain statistical significance (Fig. 3b). No differences were observed in CRP levels between the obese and non-obese groups (Fig. 3c). Also, no significant differences in plasma concentrations of PCT, ferritin and d-dimer were observed between both groups (Supplementary Fig. 3). Finally, no between-group differences in any of the ventilation parameters were observed (Supplementary Fig. 4).

\section{Clinical outcomes}

On day 40 of ICU admission, $11 \%(n=2)$ of the patients of the obese group was still mechanically ventilated and $17 \%$ $(n=3)$ was still in the ICU, while $16 \%(n=8)$ of patients of the non-obese group were still mechanically ventilated and in the ICU ( $p=0.60$ and $p=0.97$, respectively). Time on ventilator was $22(16-40)$ days in the obese group and 27 (14-40) days in the non-obese group $(p=0.41)$. ICU length of stay was 25 (17-40) and 29 (15-40) days in the obese and non-obese group, respectively $(p=0.53)$. Forty-day ICU mortality was $17 \%(n=3)$ in the obese group and $24 \%$ $(n=12)$ in the non-obese group $(p=0.50)$. The Kaplan-Meier curves for time on mechanical ventilation, ICU LOS and mortality are presented in Fig. 4.

\section{Discussion}

In this observational study in critically ill COVID-19 patients, we observed that, while disease severity and other baseline covariates were similar, temperature at ICU admission was lower in obese compared to non-obese patients. This observation may suggest that the endogenous immune-hypothalamus axis responsible for the induction of 
Fig. 1 Body mass index distribution and plasma cytokine levels. a Histogram depicting body mass index (BMI) frequencies. Kinetics of concentrations of circulating $\mathbf{b}$ interleukin (IL)-6, c IL-8, d IL-

10 , e tumor necrosis factor alpha

$(\mathrm{TNF}-\alpha), \mathbf{f}$ interferon gamma

(IFN- $\gamma$ ), $\mathbf{g}$ interferon gammainduced protein (IP)-10, $\mathbf{h}$ monocyte chemoattractant protein (MCP)-1 and i IL-1 receptor antagonist (RA). Data are presented as geometric mean with $95 \%$ confidence interval.

$* p<0.05$, calculated using Sidak's post-hoc multiple comparisons tests on individual timepoints.
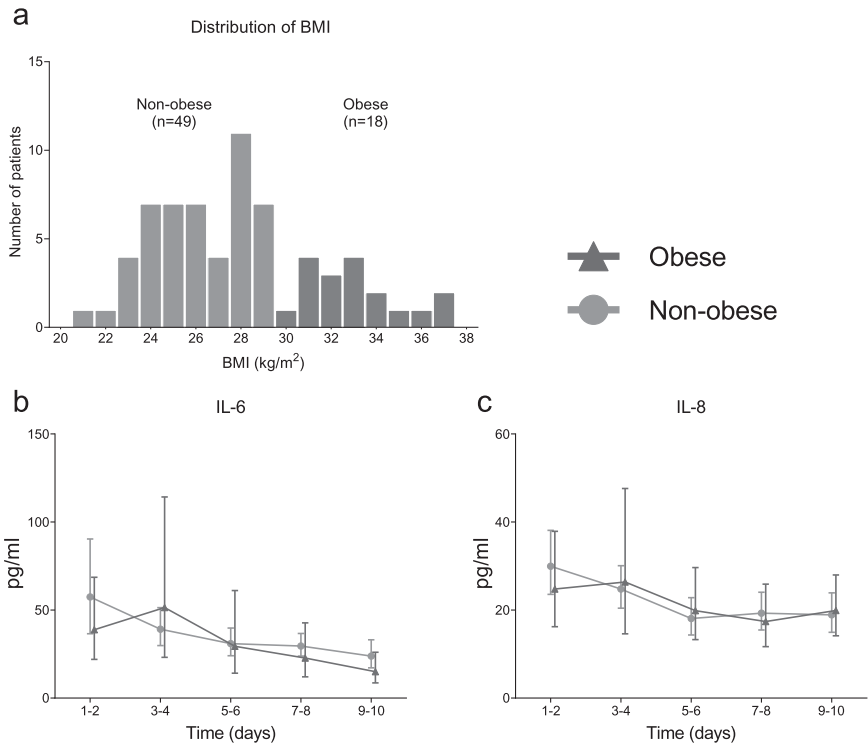

d

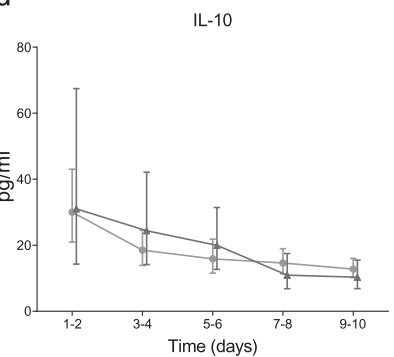

e

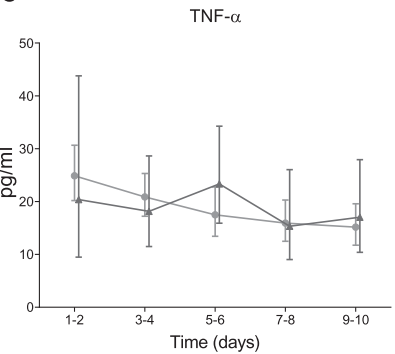

f
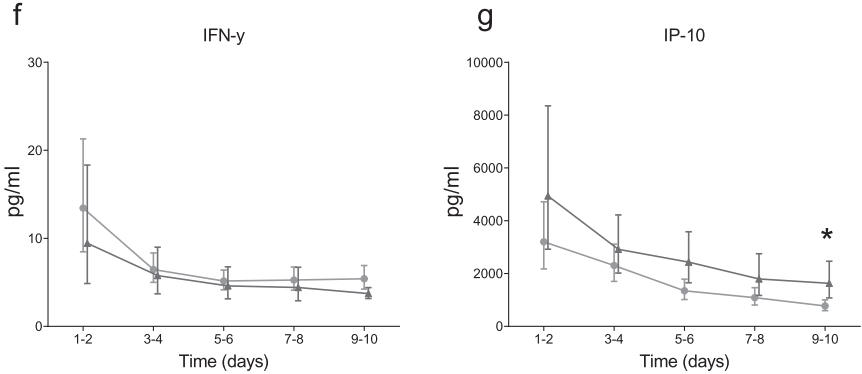

$\mathrm{h}$
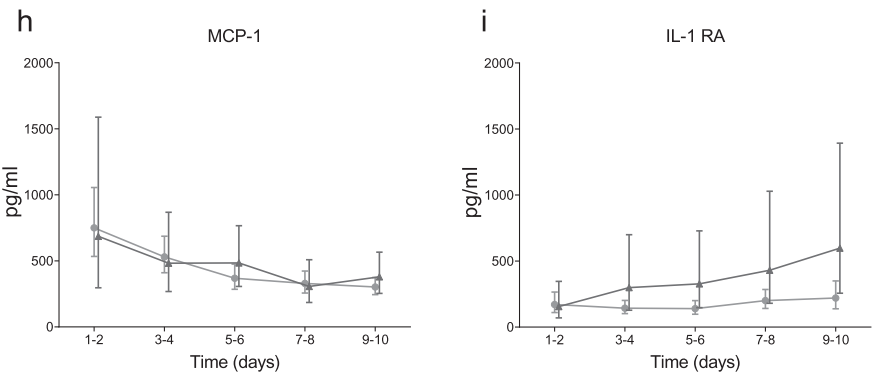

fever (IL-1-IL-6-PGE2 pathway) is less pronounced in obese compared to non-obese patients. However, no significant differences in plasma concentrations of various inflammatory markers and cytokines (most importantly IL6) were observed between both groups. To gain sensitivity to detect a possible relationship between BMI and the cytokine response we performed correlation analyses that confirmed lack of such a relationship. Also, no significant difference in duration of mechanical ventilation, ICU length of stay, or mortality was observed between the obese and non-obese group. Taken together, these data indicate that once a COVID-19 patient becomes critically ill, the immune response and clinical course of the disease is not relevantly influenced by the patients' BMI. 
Table 1 Patient characteristics at the time of ICU admission.

\begin{tabular}{|c|c|c|c|}
\hline & $\begin{array}{l}\text { Non-obese } \\
(\mathrm{BMI}<30, n=49)\end{array}$ & $\begin{array}{l}\text { Obese } \\
(\mathrm{BMI} \geq 30, n=18)\end{array}$ & $p$ value \\
\hline \multicolumn{4}{|l|}{ Characteristics } \\
\hline Body Mass Index, $\mathrm{kg} / \mathrm{m}^{2}$ & $26.0[24.4-27.7]$ & $32.6[31.2-34.5]$ & $<0.0001$ \\
\hline Age, years & $65[59-72]$ & $66.5[57-72]$ & 0.99 \\
\hline Male & $38(76 \%)$ & $12(24 \%)$ & 0.36 \\
\hline Female & $11(65 \%)$ & $6(35 \%)$ & 0.36 \\
\hline APACHE II & $14(12-19)$ & $17(9-23)$ & 0.54 \\
\hline SOFA & $7[5-8.5]$ & $4(4-9)$ & 0.72 \\
\hline Time from first COVID signs to ICU admission, days & $12[8-15.75]$ & $10[7-13.5]$ & 0.35 \\
\hline \multicolumn{4}{|l|}{ Medical history } \\
\hline Cardio vascular disease & $11(22 \%)$ & $7(39 \%)$ & 0.18 \\
\hline Hypertension & $23(47 \%)$ & $8(44 \%)$ & 0.53 \\
\hline Chronic kidney disease & $0(0 \%)$ & $1(6 \%)$ & 0.10 \\
\hline Chronic obstructive pulmonary disease & $3(6 \%)$ & $3(17 \%)$ & 0.18 \\
\hline Diabetes mellitus & $11(22 \%)$ & $4(22 \%)$ & 0.98 \\
\hline Hematological malignancy & $1(2 \%)$ & $0(0 \%)$ & 0.54 \\
\hline Metastatic neoplasm & $4(8 \%)$ & $1(6 \%)$ & 0.72 \\
\hline \multicolumn{4}{|l|}{ Clinical and laboratory parameters } \\
\hline Mean arterial pressure, $\mathrm{mmHg}$ & $90[85-94]$ & 84 [78-94] & 0.07 \\
\hline Heart rate, beats/min & 86 [77-98] & 88 [81-94] & 0.98 \\
\hline Temperature, ${ }^{\circ}$ Celsius & $38.7[38.0-39.5]$ & $38.1[36.9-38.9]$ & 0.02 \\
\hline Respiratory rate, beats/min & $22(20-25)$ & $22(20-26)$ & 0.87 \\
\hline PEEP, $\mathrm{cmH}_{2} \mathrm{O}$ & $12(10-14)$ & $12(12-14)$ & 0.15 \\
\hline Tidal volume ${ }_{\mathrm{IBW}}, \mathrm{ml} / \mathrm{kg}$ & $6.4[6.0-6.8]$ & $6.4[5.9-8.2]$ & 0.78 \\
\hline $\mathrm{PaO} 2 / \mathrm{FiO}_{2}$ ratio, $\mathrm{mmHg}$ & $144[95-180]$ & $144[107-180]$ & 0.60 \\
\hline Creatinine, $\mu \mathrm{mol} / \mathrm{L}$ & $79[65-101]$ & $97[58-229]$ & 0.28 \\
\hline Bilirubin & $7.0(6-12)$ & $6.0(4-12)$ & 0.71 \\
\hline Alanine aminotransferase, U/L & $38.0[21.0-56.0]$ & $44.0[23.5-94.0]$ & 0.38 \\
\hline Aspartate aminotransferase, U/L & $52.0[37.0-67.0]$ & $55.0[31.5-63.5]$ & 0.36 \\
\hline Leukocyte count, $\times 10^{9} / \mathrm{L}$ & $8.2[6.2-10.8]$ & $8.8[0.7-10.0]$ & 0.63 \\
\hline $\mathrm{CRP}, \mathrm{mg} / \mathrm{L}$ & 218 [169-291] & 245 [136-327] & 0.43 \\
\hline Procalcitonin, $\mu \mathrm{g} / \mathrm{L}$ & $0.76[0.39-1.99]$ & $0.53[0.28-1.56]$ & 0.52 \\
\hline Ferritin, $\mu \mathrm{g} / \mathrm{L}$ & $1486[720-2265]$ & 1129 [724-2404] & 0.74 \\
\hline D-dimer, $\mathrm{ng} / \mathrm{mL}$ & $3330[1830-10370]$ & $2560[1545-2560]$ & 0.48 \\
\hline
\end{tabular}

Data are presented as $n(\%)$ or median [IQR].
Obesity has been identified as a risk factor for severe disease and ICU admission in COVID-19 patients admitted to non-intensive care hospital wards $[16,17]$. This is not necessarily in contrast with our findings, as obese individuals may be more susceptible to infection, might have a different immune response prior to becoming critically ill, and/or may require more respiratory support [6]. Although several studies have assessed associations between BMI and clinical outcomes/mortality in critically ill COVID-19 patients, conflicting findings have been reported [11-13]. As such, the relationship between obesity and mortality in these critically ill patients remains unclear. What our study adds pertaining to the role of BMI in critically ill COVID-
19 patients are data of inflammatory/immunological markers. Of interest, a paradoxical relationship between obesity and mortality has been described in the general ICU population, as well as in critically ill patients suffering from different infectious diseases, such as pneumonia and bacterial sepsis $[18,19]$. Overall, obese patients demonstrate a better survival compared to non-obese individuals, even when corrected for several covariates. This phenomenon has been coined the obesity paradox [20]. Our study was underpowered to demonstrate statistically significant differences in mortality between obese and non-obese patients, and therefore we are unable to confirm or reject the presence of the obesity paradox in COVID-19 patients. 

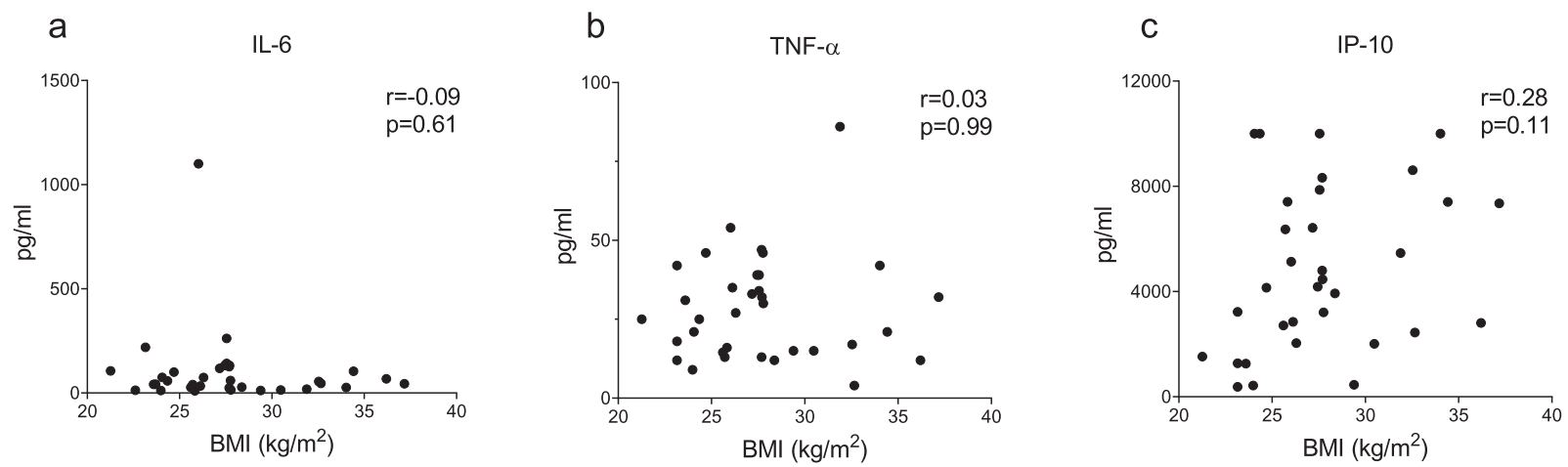

Fig. 2 Relationship between BMI and concentrations of circulating cytokines on day of admission to the intensive care unit. a Interleukin (IL)-6, b tumor necrosis factor alpha (TNF- $\alpha$ ) and $\mathbf{c}$ interferon gamma-induced protein (IP)-10. $\mathrm{r}$ - and $p$ values were calculated using

Spearman's correlation. See supplementary Fig. 2 for the relationships between BMI and the other measured circulating cytokine concentrations.
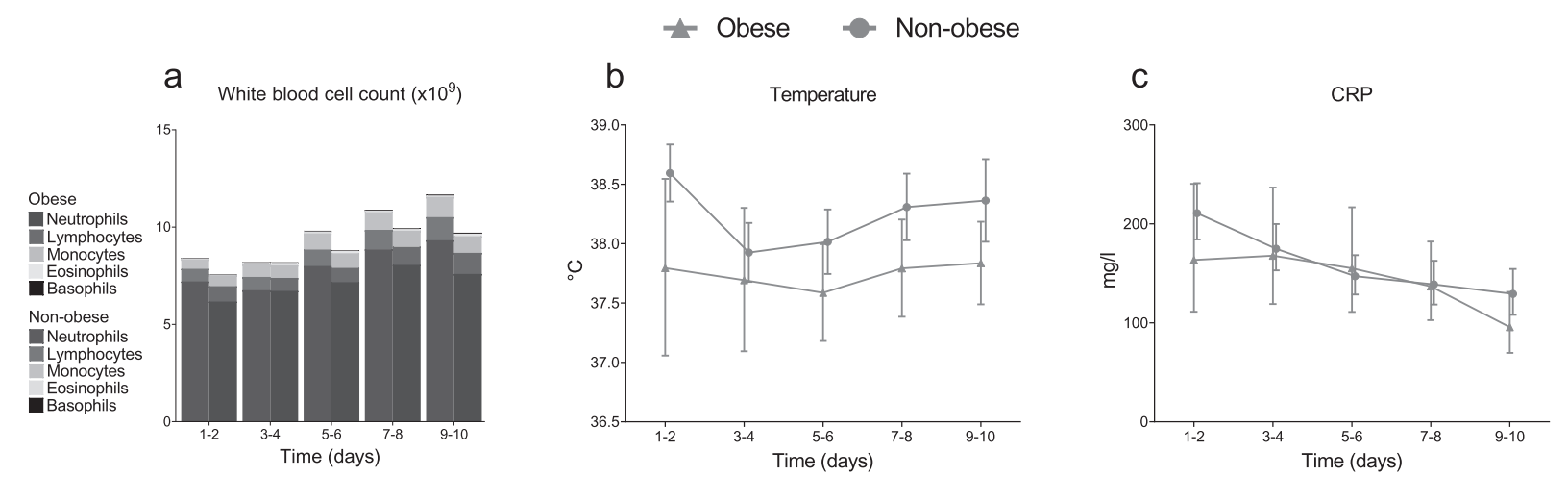

Fig. 3 Routine laboratory and clinical inflammatory parameters. a White blood cell counts (WBC), b temperature, c circulating C-reactive protein (CRP). Data are represented as geometric mean

(a) or geometric mean with $95 \%$ confidence interval (b, c). No significant differences on any of the individual timepoints were observed according to Sidak's post-hoc multiple comparisons tests.
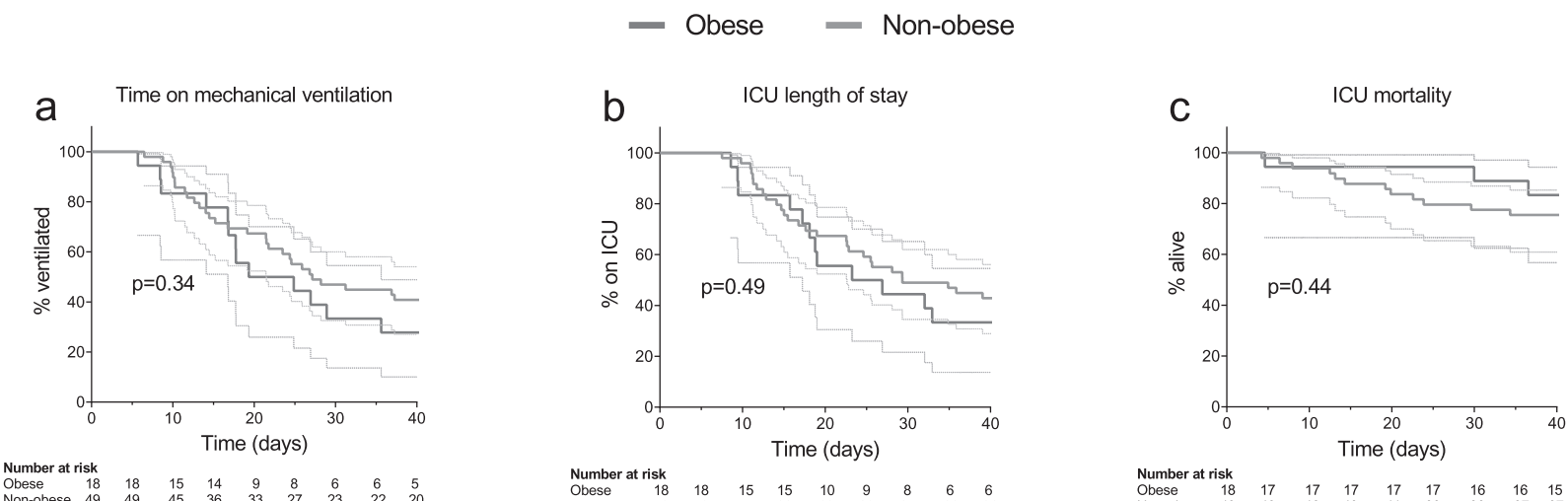

Fig. 4 Clinical outcome data. a Time on mechanical ventilation, b ICU length-of-stay, c ICU mortality. $p$ values depicted in the panels were calculated using log-rank tests. Dotted lines indicate 95\% confidence intervals.

The present study has several limitations. First, because of the observational nature of the study, no direct link between cause and effect can be deduced. Second, because of the relatively small group of (especially obese) patients, a type 2 error is possible, and our findings should be regarded as indicative, not conclusive. Nevertheless, there were also no nonsignificant trends in inflammatory parameters or correlations between BMI and any of the circulating cytokines, which would be expected in case BMI would play an crucial role in the immune response in critically ill COVID19 patients. Third, the present study was conducted in a single center in the Netherlands, where (morbid) obesity 
may be less widespread compared to other countries. Accordingly, the obese group consisted largely of mildly obese patients and no patients with a BMI above $40 \mathrm{~kg} / \mathrm{m}^{2}$ were present in our cohort. Fourth, because patients in our cohort were all included after ICU admission and received mechanical ventilation, no statements can be made about the relationship between obesity and the risk of ICU admission or requirement of mechanical ventilation. Finally, we focused on innate immunity, so possible differences between obese and non-obese COVID-19 patients in adaptive immune response remain to be explored.

In conclusion, the results of this study indicate that, in critically ill COVID-19 patients requiring mechanical ventilation, the patients' BMI is not related to a different innate immune response, unfavorable respiratory mechanics, or impaired outcome. A larger multi-center study with a more expansive BMI distribution is warranted to confirm our findings.

\section{Data availability}

The datasets generated during and/or analyzed during the current study are available from the corresponding author on reasonable request.

The RCI-COVID-19 study group Pleun Hemelaar ${ }^{2}$, Remi Beunders ${ }^{2}$, Tim Frenzel ${ }^{2}$, Jeroen Schouten ${ }^{2}$, Sjef van der Velde ${ }^{2}$, Hetty van der $\mathrm{Eng}^{2}$, Noortje Roovers', Margreet Klop-Riehl' ${ }^{2}$, Jelle Gerretsen ${ }^{2}$, Nicole Waalders ${ }^{2}$, Hidde Heesakkers ${ }^{2}$, Tirsa van Schaik ${ }^{2}$, Leonie Buijsse $^{2}$, Leo Joosten ${ }^{2}$, Quirijn de Mast ${ }^{2}$, Martin Jaeger ${ }^{2}$, Ilse Kouijzer $^{2}$, Helga Dijkstra ${ }^{2}$, Heidi Lemmers ${ }^{2}$, Reinout van Crevel $^{2}$, Josephine van de Maat ${ }^{2}$, Gerine Nijman ${ }^{2}$, Simone Moorlag ${ }^{2}$, Esther Taks ${ }^{2}$, Priya Debisarun $^{2}$, Heiman Wertheim ${ }^{2}$, Joost Hopman ${ }^{2}$, Janette RahamatLangendoen ${ }^{2}$, Chantal Bleeker-Rovers ${ }^{2}$, Hans Koenen ${ }^{2}$, Esther Fasse ${ }^{2}$, Esther van Rijssen ${ }^{2}$, Manon Kolkman ${ }^{2}$, Bram van Cranenbroek ${ }^{2}$, Ruben Smeets ${ }^{2}$, Irma Joosten ${ }^{2}$

Author contributions EJK, AHdN, WJC, MK and PP designed the study. AHdN, IG, and NAFJ were responsible for sample collection and laboratory processing. EJK, WJC, and MK were responsible for data collection and management. EJK and WJC performed the statistical analysis and drafted the manuscript. AHdN, IG, NAFJ, MGN, FLvdV, JGvdH, MK and PP critically revised the manuscript. All authors read and approved the final manuscript.

Funding The study was internally funded by the participating departments. EJK was funded by a Radboudumc COVID-19 grant. MGN was supported by an ERC Advanced Grant (\#833247) and a Spinoza Grant of the Netherlands Organization for Scientific Research.

\section{Compliance with ethical standards}

Conflict of interest The authors declare that they have no conflict of interest.

Ethical approval The study was carried out in the Netherlands in accordance with the applicable rules concerning the review of research ethics committees and informed consent. All patients or legal representatives were informed about the study details and could decline to participate.

Publisher's note Springer Nature remains neutral with regard to jurisdictional claims in published maps and institutional affiliations.

\section{References}

1. Zhou F, Yu T, Du R, Fan G, Liu Y, Liu Z, et al. Clinical course and risk factors for mortality of adult inpatients with COVID-19 in Wuhan, China: a retrospective cohort study. Lancet. 2020;395:1054-62.

2. Li X, Xu S, Yu M, Wang K, Tao Y, Zhou Y, et al. Risk factors for severity and mortality in adult COVID-19 inpatients in Wuhan. $\mathrm{J}$ Allergy Clin Immunol. 2020;146:110-8.

3. Ruan Q, Yang K, Wang W, Jiang L, Song J. Clinical predictors of mortality due to COVID-19 based on an analysis of data of 150 patients from Wuhan, China. Intensive Care Med. 2020;46:846-8.

4. Petrilli CM, Jones SA, Yang J, Rajagopalan H, O'Donnell L, Chernyak Y, et al. Factors associated with hospital admission and critical illness among 5279 people with coronavirus disease 2019 in New York City: prospective cohort study. BMJ. 2020;369: m1966.

5. Poirier P, Giles TD, Bray GA, Hong Y, Stern JS, Pi-Sunyer FX, et al. Obesity and cardiovascular disease: pathophysiology, evaluation, and effect of weight loss: an update of the 1997 American Heart Association Scientific Statement on Obesity and Heart Disease from the Obesity Committee of the Council on Nutrition, Physical Activity, and Metabolism. Circulation. 2006;113:898-918.

6. Simonnet A, Chetboun M, Poissy J, Raverdy V, Noulette J, Duhamel A. et al. High prevalence of obesity in severe acute respiratory syndrome coronavirus-2 (SARS-CoV-2) requiring invasive mechanical ventilation. Obesity. 2020;28:1195-9.

7. Hussain A, Mahawar K, Xia Z, Yang W, El-Hasani S. Obesity and mortality of COVID-19. Meta-analysis. Obes Res Clin Pract. 2020;14:295-300.

8. Mertz D, Kim TH, Johnstone J, Lam PP, Science M, Kuster SP, et al. Populations at risk for severe or complicated influenza illness: systematic review and meta-analysis. BMJ. 2013;347:f5061.

9. Littleton SW, Tulaimat A. The effects of obesity on lung volumes and oxygenation. Respir Med. 2017;124:15-20.

10. Kruglikov IL, Scherer PE. The role of adipocytes and adipocytelike cells in the severity of COVID-19 infections. Obesity. 2020;28:1187-90.

11. Auld SC, Caridi-Scheible M, Blum JM, Robichaux C, Kraft C, Jacob JT, et al. ICU and Ventilator Mortality Among Critically Ill Adults With Coronavirus Disease 2019. Crit Care Med. 2020;48: e799-804.

12. Halasz G, Leoni ML, Villani GQ, Nolli M, Villani M. Obesity, overweight and survival in critically ill patients with SARS-CoV2 pneumonia: is there an obesity paradox? Preliminary results from Italy. Eur J Prev Cardiol. 2020;1-3.

13. Gupta S, Hayek SS, Wang W, Chan L, Mathews KS, Melamed ML. et al. Factors associated with death in critically ill patients with coronavirus disease 2019 in the US. JAMA Intern Med. 2020;180:1-12.

14. World Health Organization. Obesity: preventing and managing the global epidemic. Report of a WHO consultation. World Health Organ Tech Rep Ser. 2000;894:i-xii, 1-253.

15. Brower RG, Matthay MA, Morris A, Schoenfeld D, Thompson BT, Wheeler A. Ventilation with lower tidal volumes as compared with traditional tidal volumes for acute lung injury and the acute respiratory distress syndrome. N Engl J Med. 2000;342:1301-8. 
16. Chiappetta S, Sharma AM, Bottino V, Stier C. COVID-19 and the role of chronic inflammation in patients with obesity. Int $\mathrm{J}$ Obes. 2020;44:1790-2.

17. Gao F, Zheng KI, Wang XB, Sun QF, Pan KH, Wang TY. et al. Obesity is a risk factor for greater COVID-19 severity. Diabetes Care. 2020;43:e72-4.

18. Nie W, Zhang Y, Jee SH, Jung KJ, Li B, Xiu Q. Obesity survival paradox in pneumonia: a meta-analysis. BMC Med. 2014;12:61.
19. Pepper DJ, Sun J, Welsh J, Cui X, Suffredini AF, Eichacker PQ. Increased body mass index and adjusted mortality in ICU patients with sepsis or septic shock: a systematic review and meta-analysis. Crit Care. 2016;20:181.

20. Schetz M, De Jong A, Deane AM, Druml W, Hemelaar P, Pelosi $\mathrm{P}$, et al. Obesity in the critically ill: a narrative review. Intensive Care Med. 2019;45:757-69. 